\title{
Quantification of Leukocyte Genomic 5-Methylcytosine Levels Reveals Epigenetic Plasticity in Healthy Adult Cloned Cattle
}

\author{
Béatrice de Montera, Dalia El Zeihery, ${ }^{1}$ Sigrid Müller, ${ }^{3}$ Hélène Jammes, Gottfried Brem, ${ }^{4}$ \\ Horst-Dieter Reichenbach, ${ }^{5}$ Fabian Scheipl, ${ }^{6}$ Pascale Chavatte-Palmer, Valeri Zakhartchenko, ${ }^{3}$ \\ Oliver J. Schmitz, ${ }^{2}$ Eckhard Wolf, ${ }^{3}$ Jean-Paul Renard, and Stefan Hiendleder ${ }^{3,7,8}$
}

\begin{abstract}
Successful somatic cell nuclear transfer (SCNT) requires epigenetic reprogramming of a differentiated donor cell nucleus. Incorrect reprogramming of epigenetic markings such as DNA methylation is associated with compromised prenatal development and postnatal abnormalities. Clones that survive into adulthood, in contrast, are assumed to possess a normalized epigenome corresponding to their normal phenotype. To address this point, we used capillary electrophoresis to measure 5-methylcytosine $(5 \mathrm{mC})$ levels in leukocyte DNA of 38 healthy female bovine clones that represented five genotypes from the Simmental breed and four genotypes from the Holstein breed. The estimated variance in $5 \mathrm{mC}$ level within clone genotypes of both breeds [0.104, 95\% confidence interval (CI): 0.070-0.168] was higher than between clone genotypes (0, CI: 0-0.047). We quantified the contribution of SCNT to this unexpected variability by comparing the 19 Simmental clones with 12 female Simmental monozygotic twin pairs of similar age. In Simmental clones, the estimated variability within genotype (0.0636, CI: 0.0358-0.127) was clearly higher than in twin pairs (0.0091, CI: 0.0047-0.0229). In clones, variability within genotype (0.0636) was again higher than between genotypes (0, CI: 0-0.077). Twins, in contrast, showed lower variability within genotypes (0.0091) than between genotypes (0.0136, CI: 0.00250-0.0428). Importantly, the absolute deviations of $5 \mathrm{mC}$ values of individual SCNT clones from their genotype means were fivefold increased in comparison to twins. Further comparisons with noncloned controls revealed DNA hypermethylation in most of the clones. The clone-specific variability in DNA methylation and DNA hypermethylation clearly show that healthy adult SCNT clones must be considered as epigenome variants.
\end{abstract}

\section{Introduction}

$\mathrm{C}$ LONING BY SOMATIC CELL NUCLEAR TRANSFER (SCNT) is compatible with the birth of live offspring in a wide range of mammalian species, but failure of epigenetic reprogramming leads to a low overall efficiency of the technology (Keefer et al., 2008; Yang et al., 2007a). The impact of SCNT procedures on the epigenome is thought to be stochastic, with a potential for subtle long-term epigenetic defects (Jaenisch and Wilmut, 2001; Rhind et al., 2003b; Wilmut, 2006). High rates of embryonic and fetal mortality (Rhind et al., 2003a; Yang et al., 2007a) and an increased incidence of congenital defects (Chavatte-Palmer et al. 2002; Rhind et al., 2003a, 2003b) have been linked to perturbations in developmentally

${ }^{1}$ INRA, UMR 1198 Biologie du Développement et Reproduction, Jouy en Josas, France.

${ }^{2}$ Department of Analytical Chemistry, University of Wuppertal, Wuppertal, Germany.

${ }^{3}$ Chair for Molecular Animal Breeding and Biotechnology, and Laboratory for Functional Genome Analysis (LAFUGA), Gene Center, LMU Munich, Munich, Germany.

${ }^{4}$ Institute of Animal Breeding and Genetics, University of Veterinary Sciences, Vienna, Austria.

${ }^{5}$ Bayerische Landesanstalt für Landwirtschaft, Poing/Grub, Germany.

${ }^{6}$ STABLAB, LMU Munich, Munich, Germany.

${ }^{7}$ JS Davies Epigenetics and Genetics Group, Animal Science; School of Agriculture, Food \& Wine; The University of Adelaide, Adelaide, Australia.

${ }^{8}$ Robinson Institute, Research Centre for Reproductive Health, The University of Adelaide, Adelaide, Australia. 
important epigenetic marks such as DNA methylation and histone modifications (Humpherys et al., 2001; Lin et al., 2008; Liu et al., 2008; Ohgane et al., 2001, 2004; Santos et al., 2003). Clones that develop into adults have nevertheless been assumed to possess a normalized epigenome that corresponds to their normal phenotype (Lanza et al., 2001; Senda et al., 2007).

Methylation of cytosine residues at $\mathrm{CpG}$ dinucleotides (5-methylcytosine or $5 \mathrm{mC}$ ) is the most extensively studied epigenetic modification in mammals and is essential for regulating embryonic development, transcription, chromatin structure, $\mathrm{X}$ chromosome inactivation, genomic imprinting, and chromosome stability (Robertson, 2005). The genomewide quantification of $5 \mathrm{mC}$ levels in SCNT cloned embryos from different species has revealed aberrations in global DNA methylation (Dean et al., 2001; Yang et al., 2007c). In cattle, which are used extensively as a model for mammalian cloning (Yang et al., 2007a), an increased genome-wide DNA methylation level, that is, DNA hypermethylation, has been correlated with poor developmental potential of preimplantation embryos (Santos et al., 2003) and fetal overgrowth (Hiendleder et al., 2004). Abnormal DNA methylation patterns in clones have also been described for specific genes and noncoding DNA sequences under epigenetic control (Bourc'his et al., 2001; Lin et al., 2008; Liu et al. 2008; Morgan et al., 2005; Niemann et al., 2008; Yang et al., 2007a). Despite these reports, the majority of cloned cattle do not present health issues of concern beyond a critical period of about 6 months after birth and are assumed to be normal (Lanza et al., 2001; Wells et al., 2004; Yang et al., 2007b). However, the DNA methylation status of these apparently healthy adult cattle clones has not, to our knowledge, been investigated. This reflects the general assumption that clones surviving into adulthood should have a DNA methylation level very similar to individuals that originate from sexual reproduction.

In this study, we used highly sensitive capillary electrophoresis to quantify the genomic $5 \mathrm{mC}$ content of DNA from healthy adult female cattle clones in comparison with noncloned controls. The analyses revealed an unexpected epigenetic plasticity of healthy adult clones, suggesting that epigenome variability may be representative of adaptive changes after reproductive cloning.

\section{Materials and Methods}

\section{SCNT and twin animals}

SCNT cloned animals were generated by two standard SCNT procedures (Bourc'his et al., 2001; Hiendleder et al., 2004; Vignon et al., 1998; Zakhartchenko et al., 1999) with fibroblasts from nine donors that yielded two to nine clones per donor. Embryos were transferred to synchronous recipients at the blastocyst stage. Only grade 1 and 2 embryos according to the morphological criteria defined by the International Embryo Transfer Society (www.iets.org) were used. Monozygotic twins were generated by microsurgical bisection of fertilised embryos at the blastocyst stage (Klein et al., 2006). Within each of the two breeds used, all animals (clones and donor or clones and twins) were subjected to the same breeding conditions and raised in the same experimental farm.

No animal was specifically generated for the present study. All animal experiments were carried out in accor- dance with European legislation on animal ethics and welfare.

\section{DNA samples}

Single 10-mL EDTA blood samples were obtained by jugular vein puncture for each animal and leukocyte separation was performed by centrifugation at 1,200 $\times g, 25 \mathrm{~min}$. The leukocyte fraction was washed with $10 \mathrm{~mL}$ of phosphatebuffered saline (PBS) and further centrifuged at $530 \times g$, $10 \mathrm{~min}$. Cell counts were performed for $1 \mathrm{~mm}^{3}$ of nondiluted aliquot per sample, using the Malassey cell counting method. The remaining leukocyte fraction was used for DNA extraction with DNeasy Blood \& Tissue kit from Qiagen (Chatsworth, CA, USA). DNA samples were dissolved in distilled water and stored at $-20^{\circ} \mathrm{C}$.

\section{Micellar electrokinetic chromatography (MEKC)}

We used a highly sensitive improved capillary electrophoresis technique (Schmitz et al., 2002; Stach et al., 2003) to measure the $5 \mathrm{mC}$ content in leukocyte DNA of clones and twins. One microgram of dry genomic DNA sample was hydrolyzed to $2^{\prime}$ deoxynucleoside-3'-monophosphate. The DNA hydrolysates were derivatized with Bodipy FL EDA fluorescent marker. The surplus of Bodipy was precipitated and the aqueous phase was analyzed by MEKC using $\mathrm{P} / \mathrm{ACE}^{\mathrm{TM}} \mathrm{MDQ}$ system with a laser-induced fluorescence detector (Beckman Coulter, Fullerton, CA, USA) and a fused silica capillary $\left(50 \mu \mathrm{m} \mathrm{ID,} \mathrm{L}_{\text {tot }}=50 \mathrm{~cm}\right.$, detection window at $\mathrm{L}_{\text {eff }}=40 \mathrm{~cm}$ ) (BGB-Analytik, Geneva, Switzerland). Nucleotide separation was performed using sodium phosphate buffer containing SDS solution and $20 \mathrm{kV}$ voltage. From 7 to 15 capillary electrophoresis runs per sample were performed and further analyzed with $32 \mathrm{Karat}^{\mathrm{TM}}$ Software, Version 7 (Beckman Coulter). Standard deviations of mean $5 \mathrm{mC}$ values were below $5 \%$.

\section{Statistical methods}

We ran separate models for the three experimental groups to estimate interindividual and intergenotype variances and another linear mixed effects model for the combined measurements of all Simmental animals to estimate the difference in average methylation levels between clones and twins. Data analysis was carried out with the statistical software environment R 2.9.2 (R Development Core Team, 2008). Specifically, we used the lme4 package (Bates, 2009) for the estimation of the linear mixed effects models (Pinheiro and Bates, 2000) and the RLRsim package (Scheipl, 2007) to compute exact restricted likelihood ratio tests (Scheipl et al., 2008) for the presence of between-genotype variation. Reported $p$-values for the latter are based on Monte Carlo samples of 10,000 values from the exact finite sample distribution of the likelihood ratio. Confidence intervals (CI) for the estimated variances are $95 \%$ profile likelihood intervals.

\section{Results}

\section{Genomic DNA methylation levels in clones} and controls

We measured and analyzed genomic $5 \mathrm{mC}$ content of leukocyte DNA from 38 healthy adult female SCNT clones 
that represented four genotypes of the Holstein breed and five genotypes of the Simmental breed. Experimentally generated monozygotic Simmental twins and Holstein nuclear donor animals served as controls (Fig. 1A). We observed $5 \mathrm{mC}$ levels that ranged from $4.43-5.69 \%$ in clones with Holstein genetics $(n=19)$ to $5.94-6.93 \%$ in clones with Simmental genetics $(n=19)$. The $5 \mathrm{mC}$ levels of controls ranged from $4.56-5.09 \%$ in Holstein nuclear donor animals $(n=3)$ and from $5.07-5.64 \%$ in Simmental monozygotic twins $(n=24)$.

ANOVA showed significant differences in the mean $5 \mathrm{mC}$ content of DNA between Holstein and Simmental clones $(6.50 \pm 0.01 \%$ SEM and $5.09 \pm 0.02 \%$ SEM, respectively; twotailed $t$-test $p<0.001)$.

\section{Genomic DNA methylation variation in clones and controls}

The observed variances between clone genotypes of both breeds were similar (0.0204 and 0.0164) and we computed exact restricted likelihood ratio tests on the basis of linear mixed effects models to test for the presence of variability between genotypes. Strikingly, we found that the estimated variance in $5 \mathrm{mC}$ level within clone genotypes from both breeds (0.104, CI: 0.070-0.168) was higher than the estimated variance between clone genotypes, which was not significantly different from 0 (exact restricted likelihood ratio test $p>0.999$, estimated value 0 with CI: $0-0.077$ ).

A

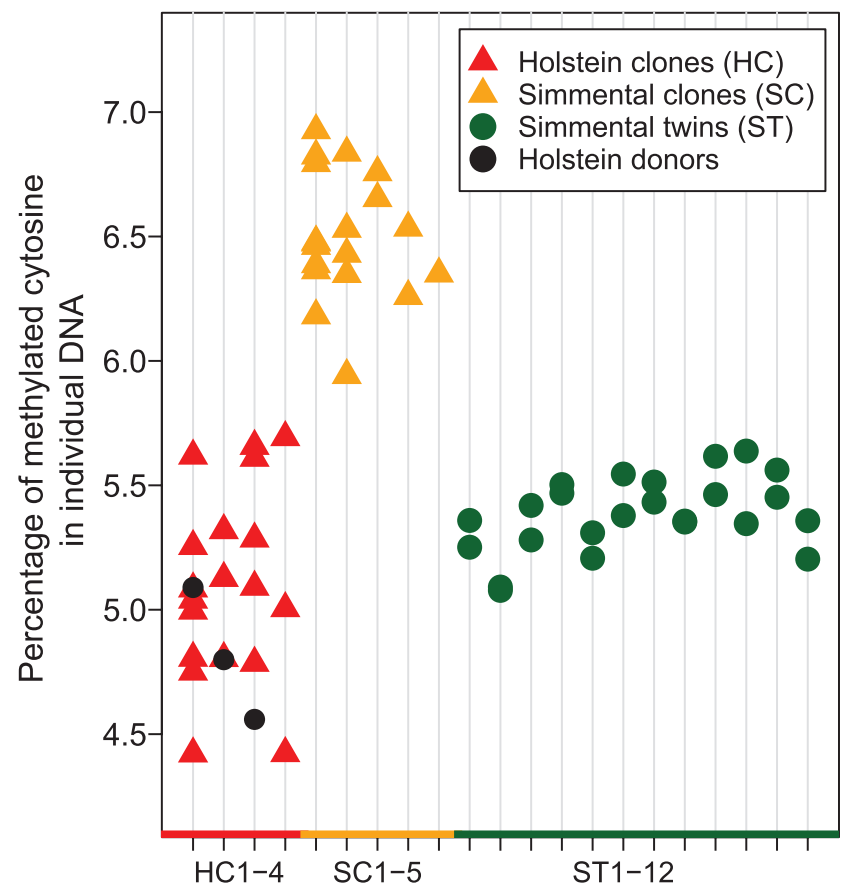

There was no correlation between the proportions of leukocyte subfractions and genomic methylation levels, excluding the possibility that the observed variability in DNA methylation levels was caused by variation in leukocyte subfractions.

\section{Genomic DNA methylation variation in clones and monozygotic twins}

To quantify the contribution of SCNT to the observed variability between clones, we compared Simmental clones with female Simmental monozygotic twins of similar age (65.5 months \pm 3.6 months SEM and 67.8 months \pm 2.2 months SEM) (Fig. 1A-C). The monozygotic twins were generated by microsurgical bisection of fertilised embryos (Klein et al., 2006). The estimated variability of $5 \mathrm{mC}$ levels within five genotypes of clones $(0.0636, n=19$, CI: 0.0358 $0.127)$ was clearly higher than in 12 twin pairs $(0.0091, n=24$, CI: 0.0047-0.0229). In Simmental clones, the estimated variability within genotype (0.0636) was higher than between ( 0 , CI: $0-0.077)$ clone genotypes. In contrast, the variability within twin genotypes (0.0091) was lower than between twin genotypes (0.0136, CI: 0.0025-0.0428), as expected (observed variance ratio: 1.485 , CI: 0.992-1.612). Importantly, the absolute deviations of $5 \mathrm{mC}$ values of individual SCNT clones from their genotype means were fivefold increased in comparison to twins, although the deviations of genotype means from the respective group means were similar for clones and
B

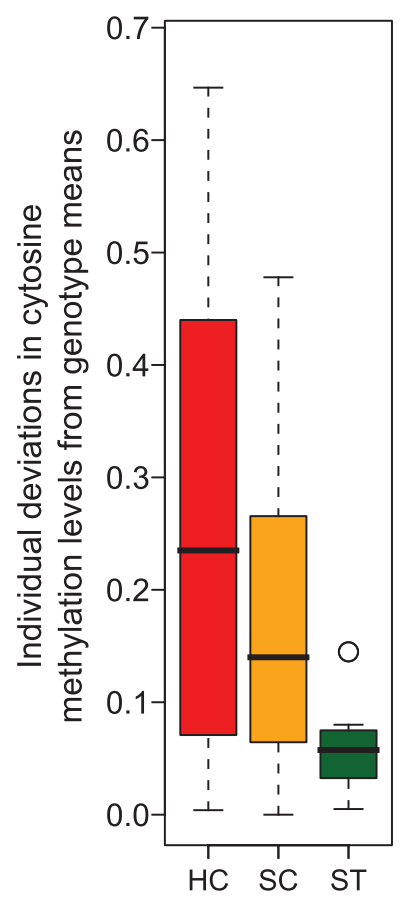

C

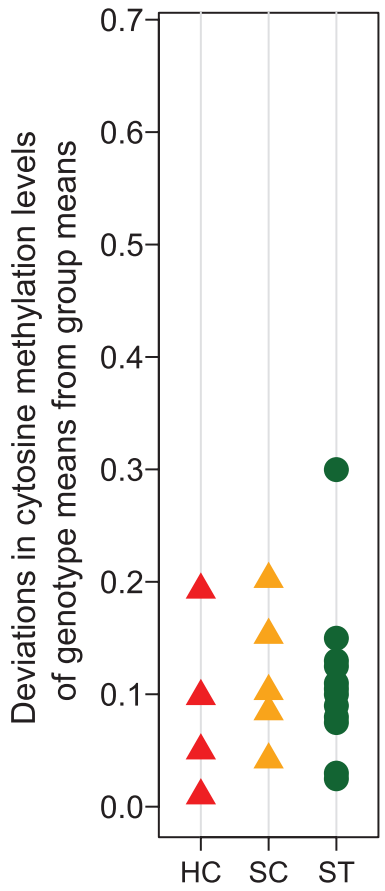

FIG. 1. Variability in genomic cytosine methylation $(5 \mathrm{mC})$ levels of healthy adult bovine SCNT clones and monozygotic twins. (A) $5 \mathrm{mC}$ in leukocyte DNA from individual Holstein breed clones (genotypes HC1-4), Simmental breed clones (genotypes SC1-5), and Simmental breed twins (genotypes ST1-12). Nuclear donors for clone genotypes HC1-3 are indicated. (B) Box plots of the absolute deviations of individual Holstein clones (HC), Simmental clones (SC), and monozygotic Simmental twins (ST), from their respective genotype means. Median (horizontal line), minimum and maximum of the distribution (vertical lines) are indicated. (C) Dot plots of the absolute deviations of genotype means for Holstein clone genotypes, Simmental clone genotypes, and Simmental twin pairs, from their respective group mean. 
twins (Fig. 1B and C). This difference in absolute deviations of individual $5 \mathrm{mC}$ values from genotype means was even more pronounced in Holstein clones (Fig. 1B and C). These methylation differences were not due to differences in animal age at sampling as shown by regression analysis (Fig. 2).

\section{Genomic DNA hypermethylation in clones}

Three Holstein individuals that served as nuclear donors were analyzed for the $5 \mathrm{mC}$ content of their genome together with their clones. Two nuclear donors exhibited the lowest $5 \mathrm{mC}$ levels of the respective genotypes. The $5 \mathrm{mC}$ level of the third nuclear donor was identical to only one of its eight healthy clones but higher than in five of them and lower than in the two remaining ones (Fig. 1A).

We detected a general DNA hypermethylation in Simmental clones compared with Simmental twins $(6.50 \pm 0.01 \%$ SEM and $5.38 \pm 0.01 \%$ SEM, two-tailed $t$-test $p<0.001)$. The clone-twin comparison extended the previously observed global DNA hypermethylation in embryonic (Dean et al., 2001) and fetal SCNT stages (Hiendleder et al., 2004) to healthy adults.

\section{Discussion}

Global DNA methylation reprogramming during preimplantation development presents a window for epigenetic perturbation that may affect gene expression and phenotype throughout pre- and postnatal life (Morgan et al., 2005). The reprogramming process establishes first the fully totipotent state and then the pluripotent state in the embryo; changes in gene expression during development are associated with changes in epigenetic modifications. The methylation of DNA is involved in long-term epigenetic silencing of specific sequences, including transposons, imprinted genes, and pluripotency-associated genes (Reik, 2007). This process could be altered through nuclear transfer procedures, and perturbed DNA methylation patterns are assumed to be responsible for changes in gene expression of compromised

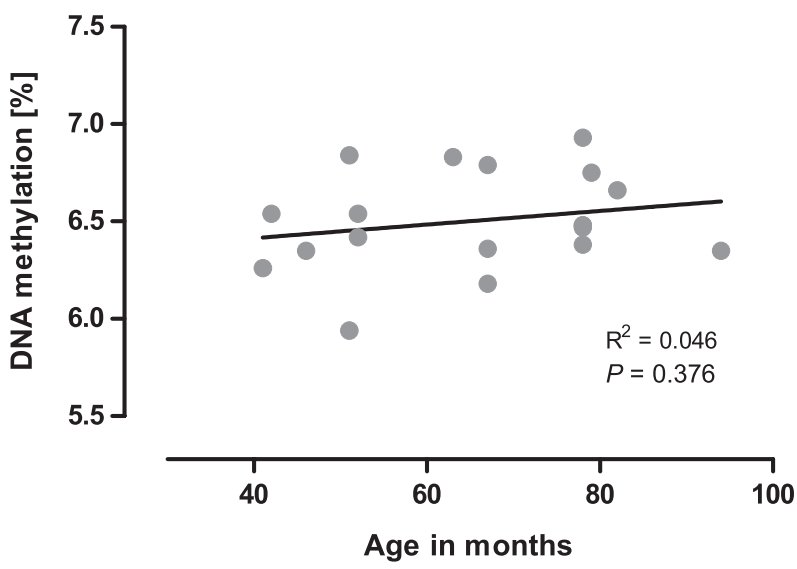

FIG. 2. Absence of age-related effects on genomic cytosine methylation $(5 \mathrm{mC})$ levels of SCNT clones. Regression analysis of genomic DNA methylation level (\% 5mC) in leukocyte DNA from individual Simmental breed clones $(n=19)$ on age of the same animals. Regressions obtained for Holstein breed clones $(n=19)$ and Simmental breed twins $(n=24)$ were also nonsignificant (data not shown). cloned embryos, fetuses, and nonviable offspring (Dean et al., 2001; Lin et al., 2008; Yang et al., 2007a).

Individual genome-wide $5 \mathrm{mC}$ levels in leukocytes were recently shown to be similar in adult humans across populations (Axume et al., 2007). Our comparison of adult cloned animals with their adult nuclear donor, and the comparison between clones and experimentally generated monozygotic twins of similar age, provides an estimate of global DNA methylation variability in leukocytes of clones. This epigenetic plasticity is currently being confirmed in liver tissue from the same Holstein clones, where first measurements of one genotype revealed individual $5 \mathrm{mC}$ levels of $3.98 \pm 0.08 \%$ SD to $4.83 \pm 0.11 \%$ SD in clones, and a value of $4.04 \pm 0.08 \%$ $\mathrm{SD}$ for the donor cow. The comparison of clones with twins confirms a SCNT cloning effect illustrated by a clone-specific variability of global DNA methylation. These epigenome variants clearly show that clones are only genomic copies (Campbell, 1999), but not epigenomic copies, of adult animals. It is currently not known if the observed methylation differences in compromised clones result from incomplete epigenetic reprogramming of the early SCNT embryo or other stochastic perturbations that affected maintenance methylation during later developmental stages (Liu et al., 2008; Niemann et al., 2008). Our study of DNA methylation levels in adult clones contributes new insights to this question by showing that variability in DNA methylation levels in live clones is not correlated with the potential to provide consistently full-term reprogramming into live calves. This is illustrated by the comparison between $\mathrm{HC} 1$ and $\mathrm{HC} 2$ genotypes that displayed individual $5 \mathrm{mC}$ levels from $4.43 \pm$ $0.04 \%$ SD to $5.61 \pm 0.13 \%$ SD with $38.8 \%$ blastocysts (per fused embryos) and $8.2 \%$ born calves (per transferred embryos), and from $4.81 \pm 0.05 \%$ SD to $5.31 \pm 0.06 \%$ SD with $35.6 \%$ blastocysts and $2.4 \%$ born calves, respectively (Fig. $1 \mathrm{~A})$. We can therefore conclude that the observed methylation variability in adult clones is independent from the breed and is not necessarily correlated with the potential of full-term development.

The DNA methylation levels in the great majority of clones were also higher than in the nuclear donor animals or monozygotic twins. In the Simmental breed, we detected a $25 \%$ increase in DNA methylation levels of clones in comparison with twins. The SCNT procedure has been shown to impact on the DNA methylation status of cloned embryos (Dean et al., 2001; Santos et al., 2003) where centromeric heterochromatin remains methylated at a higher than normal level (Bourc'his et al., 2001). DNA hypermethylation in liver has been associated with fetal overgrowth (Hiendleder et al., 2004). Further, hypermethylation of imprinted genes has been associated with perinatal death of clones (Lin et al., 2008). Our observations provide further evidence that reprogramming of nuclear function by SCNT, which is frequently associated with global DNA hypermethylation, might be maintained from the embryonic (Dean et al., 2001; Yang et al., 2007c) and fetal stages (Hiendleder et al., 2004) into adulthood.

Epigenetic differences in monozygotic twins can be explained by external factors such as different environmental exposure that causes differences in physiological activities, and internal factors such as epigenetic transmission defects through cell divisions. The latter lead to an accumulation of epigenetic differences by "epigenetic drift" associated with aging, as observed in monozygotic twins in human (Fraga 
et al., 2005). Interestingly, our data suggest that in clones the aging process and associated epigenetic drift could act differently. Regression analyses of clone data did not reveal any significant association between DNA methylation level and age (Fig. 2). Further, DNA hypermethylation is unlikely to be age-related in clones because the age of Holstein and Simmental clones overlapped and the mean ages of some genotypes such as Simmental clone genotype SC3 (80.5 months \pm 1.5 months SEM) and Holstein clone genotype HC4 (72.0 months \pm 5.8 months SEM) were similar. The significant differences in DNA methylation levels between Holstein and Simmental clones could stem from different parameters such as breed-specific differences in poorly understood reprogramming efficiency, donor cell preparation, SCNT procedures and possible stochastic effects of the whole process. The comparison between LMU and INRA cloning procedures (Bourc'his et al., 2001; Hiendleder et al., 2004) shows several differences. In the LMU cloning procedure, donor cells are subconfluent when used for nuclear transfer, whereas they are quiescent in the INRA protocol. After fusion between the donor cell and the recipient oocyte, the activation process is different and delayed for $1 \mathrm{~h}$ and a half in the LMU compared to the INRA protocol. Finally, embryo culture conditions in the LMU and INRA protocols were different, that is, SOF medium $+10 \%$ serum and $\mathrm{O}_{2}$ control for the LMU clones and B2 medium $+2.5 \%$ serum and no $\mathrm{O}_{2}$ control for INRA clones. This is reported to impact on sequence-specific methylation status and developmental gene expression in mammals (Fauque et al., 2009). Therefore, we hypothesize that SCNT induces an experimental protocol dependent "hypermethylation drift" in global DNA methylation levels that is within health compatible limits and potentially confounded with breed-specific effects, when two different breeds are compared. In the future, it will be interesting to compare the success rate of different cloning protocols with respect to healthy animals and their DNA methylation levels and to evaluate the possibility of a breedspecific susceptibility to technical constraints by testing the same conditions in different breeds.

Moreover, our data do not confirm a normalization of DNA methylation differences between clones and nonclones with advancement of age, as suggested by a study with a limited number of SCNT cloned mice (Senda et al., 2007). Instead, our data support the alternative hypothesis discussed by Senda et al. (2007) that only cloned animals with a more appropriate methylation status can survive to adulthood. This assumption is further supported by data from gene-specific analyses that revealed more severely altered epigenetic marks in clones that died soon after birth than in animals that died as juveniles (Lin et al., 2008).

In healthy adult clones, epigenetic marks that are known to be required for developmental outcome should have been maintained. We analyzed target-sequences that were previously reported to be affected by SCNT (Lin et al., 2008) and found no significant differences in the DNA methylation percentage at specific sequences (data not shown) in leukocyte DNA of the investigated Holstein clones $(n=19)$. However, sequence-specific variations in DNA methylation were observed in cloned cattle that died around birth (Long and Cai, 2007). Here, variable and altered DNA methylation patterns were detected in an imprinting control region (ICR), but not in the $3^{\prime}$-UTR region of the same locus. Studying global $5 \mathrm{mC}$ variations does not allow identification of differentially methylated regions in the genome, which constitutes a clear limitation when analyzing the potential effect of such variations on physiology and phenotype. Yet, measuring the $5 \mathrm{mC}$ amount in the whole genome is a way to include the potential effect of methylation deregulation in non coding regions. In the present study, the observed global $5 \mathrm{mC}$ variations of up to $25 \%$ could not only stem from the limited number of differentially methylated imprinted genes or regions (Luedi et al., 2005) but must involve other sequence elements such as transposons or satellite DNA. There are currently significant gaps in our understanding of the causes of epigenetic change in healthy adult cloned animals that call for further genome-wide analyses of candidate methylated sequences.

Cloning by SCNT is compatible with the birth of live offspring in a wide range of mammalian species, but failure of epigenetic reprogramming leads to a low overall efficiency of the technology. The epigenetic status of SCNT clones that survive into adulthood was nevertheless assumed to be normal. Considering the crucial roles of DNA methylation in the regulation of fundamental cellular processes and transgenerational effects (Bernstein et al., 2007; Richards, 2006), we evaluated long-term effects of SCNT reprogramming by quantifying global epigenetic plasticity in adult healthy cloned cattle. In conclusion, our data highlight SCNT effects on DNA methylation of healthy adult clones that are resilient to epigenetic reprogramming during development and postnatal exposure to environmental effects. We demonstrate here that, through SCNT cloning procedures, functional reprogramming of a donor genome into healthy adults is compatible with a highly flexible methylation status of its DNA.

Recent data obtained from monozygotic twin studies have highlighted the important role of epigenome variations as the basis for differences in heritable complex traits (Kaminsky et al., 2009) and new epigenome perspectives show the dynamic interplay of chromatin and DNA sequence in complex trait heritability (Johannes et al., 2008). The present study provides experimental support for the potential of using sets of adult cloned animals as research models for an in-depth readout of the contribution of epigenetics to the variability of complex phenotypic traits. The potential biological relevance of the unexpected epigenetic plasticity of healthy adult clones awaits further examination in view of the likely dissemination of cloned (epi)genetics into domestic animal populations in the near future (Fox, 2008).

\section{Author's Contributions}

S.H., E.W., J.P.R., O.J.S., and B.dM. conceived and designed the experiments. D.EZ., B.dM., S.M., P.C.P., and G.B. performed the experiments. S.M., F.S., E.W., B.dM., S.H., and H.J. analyzed the data. B.dM., P.C.P., S.M., V.Z., D.E.Z., O.J.S., H.D.R., and F.S. contributed reagents/materials/ analysis tools. B.dM., S.H., J.P.R., E.W., and H.J. wrote the manuscript. All authors commented on manuscript drafts.

\section{Acknowledgments}

This study was supported by the European Commission FP6 contract No. FOOD-CT-2006-016250 ("SABRE"), by the Deutsche Forschungsgemeinschaft, by the Egyptian mission and Deutscher Akademischer Austausch Dienst (DAAD), by 
the French National Institute of Agronomical Research (INRA) and by a PhD grant from the French Ministry of Research (MRES) to B.dM. S.H. is a J.S. Davies Fellow. We thank X. Vignon for advice on cloning procedures and D. Khan for advice on the manuscript. The authors acknowledge Y. Heyman, C. Richard, P. Laigre, P. Rieblinger, and all members of INRA experimental Center of UCEA Bressonvilliers and the Moorversuchsgut Badersfeld for technical support and animal care.

\section{Author Disclosure Statement}

The authors declare that no conflicting financial interests exist.

\section{References}

Axume, J., Smith, S.S., Pogribny, I.P., et al. (2007). Global leukocyte DNA methylation is similar in African American and Caucasian women under conditions of controlled folate intake. Epigenetics 2, 66-68.

Bates, D. (2009). lme4: linear mixed-effects models using S4 classes. R package version 0.999375-31.

Bernstein, B.E., Meissner, A., and Lander, E.S. (2007). The mammalian epigenome. Cell 128, 669-681.

Bourc'his, D., Le Bourhis, D., Patin, D., et al. (2001). Delayed and incomplete reprogramming of chromosome methylation patterns in bovine cloned embryos. Curr. Biol. 11, 1542-1546.

Campbell, K. (1999). Nuclear transfer in farm animal species. Semin. Cell Dev. Biol. 10, 245-252.

Chavatte-Palmer, P., Heyman, Y., Richard, C., et al. (2002). Clinical, hormonal and hematologic characteristics of bovine calves derived from nuclei from somatic cells. Biol. Reprod. 66, 1593-1603.

Dean, W., Santos, F., Stojkovic, M., et al. (2001). Conservation of methylation reprogramming in mammalian development: aberrant reprogramming in cloned embryos. Proc. Natl. Acad. Sci. USA 98, 13734-13738.

Fauque, P., Jouannet, P., Lesaffre, C., et al. (2009). Assisted reproductive technology affects developmental kinetics, H19 imprinting control region methylation and H19 gene expression in individual mouse embryos. BMC Dev. Biol. 7, 116134.

Fox, J.L. (2008). Cloned animals deemed safe to eat, but labelling issues loom. Nat. Biotechnol. 26, 249-250.

Fraga, M.F., Ballestar, E., Paz, M.F., et al. (2005). Epigenetic differences arise during the lifetime of monozygotic twins. Proc. Natl. Acad. Sci. USA 102, 10604-10609.

Hiendleder, S., Mund, C., Reichenbach, H.D., et al. (2004). Tissuespecific elevated genomic cytosine methylation levels are associated with an overgrowth phenotype of bovine fetuses derived by in vitro techniques. Biol. Reprod. 71, 217-223.

Humpherys, D., Eggan, K., Akutsu, H., et al. (2001). Epigenetic instability in ES cells and cloned mice. Science 293, 95-97.

Jaenisch, R., and Wilmut, I. (2001). Developmental biology: don't clone humans! Science 291, 2552.

Johannes, F., Colot, V., and Jansen, R.C. (2008). Epigenome dynamics: a quantitative genetics perspective. Nat. Rev. Genet. 9, 883-890.

Kaminsky, Z.A., Tang, T., Wang, S.C., et al. (2009). DNA methylation profiles in monozygotic and dizygotic twins. Nat. Genet. 41, 240-245.

Keefer, C.L. (2008). Lessons learned from nuclear transfer (cloning). Theriogenology 69, 48-54.
Klein, C., Bauersachs, S., Ulbrich, S.E., et al. (2006). Monozygotic twin model reveals novel embryo-induced transcriptome changes of bovine endometrium in the preattachment period. Biol. Reprod. 74, 253-264.

Lanza, R.P., Cibelli, J.B., Faber, D., et al. (2001). Cloned cattle can be healthy and normal. Science 294, 1893-1894.

Lin, L., Li, Q., Zhang, L., et al. (2008). Aberrant epigenetic changes and gene expression in cloned cattle dying around birth. BMC Dev. Biol. 8, 14-23.

Liu, J.H., Yin, S., Xiong, B., et al. (2008). Aberrant DNA methylation imprints in aborted bovine clones. Mol. Reprod. Dev. 75, 598-607.

Long, J.E., and Cai, X. (2007). Igf-2r expression regulated by epigenetic modification and the locus of gene imprinting disrupted in cloned cattle. Gene 388, 125-134.

Luedi, P.P., Hartemink, A.J., and Jirtle, R.L. (2005). Genomewide prediction of imprinted murine genes. Genome Res. 15, 875-884.

Morgan, H.D., Santos, F., Green, K., et al. (2005). Epigenetic reprogramming in mammals. Hum. Mol. Genet. 14(Spec. No. 1), R47-R58.

Niemann, H., Tian, X.C., King, W.A., et al. (2008). Epigenetic reprogramming in embryonic and foetal development upon somatic cell nuclear transfer cloning. Reproduction 135, 151-163.

Ohgane, J., Wakayama, T., Kogo, Y., et al. (2001). DNA methylation variation in cloned mice. Genesis 30, 45-50.

Ohgane, J., Wakayama. T., Senda, S., et al. (2004). The Sall3 locus is an epigenetic hotspot of aberrant DNA methylation associated with placentomegaly of cloned mice. Genes Cells 9, 253-260.

Pinheiro, J., and Bates, D. (2000). Mixed-Effects Models in $S$ and S-Plus. (Springer, Berlin).

R Development Core Team. (2008). R: A Language and Environment for Statistical Computing ( $\mathrm{R}$ Foundation for Statistical Computing, Vienna, Austria). Available at: http://www .R-project.org

Reik, W. (2007). Stability and flexibility of epigenetic gene regulation in mammalian development. Nature 447, 425-432.

Rhind, S.M., King, T.J., Harkness, L.M., et al. (2003a). Cloned lambs-lessons from pathology. Nat. Biotechnol. 21, 744-745.

Rhind, S.M., Taylor, J.E., De Sousa, P.A., et al. (2003b). Human cloning: can it be made safe? Nat. Rev. Genet. 4, 855-864.

Richards, E.J. (2006). Inherited epigenetic variation-revisiting soft inheritance. Nat. Rev. Genet. 7, 395-401.

Robertson, K.D. (2005). DNA methylation and human disease. Nat. Rev. Genet. 6, 597-610.

Santos, F., Zakhartchenko, V., Stojkovic, M., et al. (2003). Epigenetic marking correlates with developmental potential in cloned bovine preimplantation embryos. Curr. Biol. 13, 1116-1121.

Scheipl, F. (2007). RLRsim: restricted likelihood ratio tests in linear mixed models. R package version 2007, 1.0.

Scheipl, F., Greven, S., and Küchenhoff, H. (2008). Size and power of tests for a zero random effect variance or polynomial regression in additive and linear mixed models. Comput. Stat. Data Anal. 52, 3283-3299.

Schmitz, O.J., Wörth, C.C.T., Stach, D., et al. (2002). Capillary electrophoresis analysis of DNA adducts as biomarkers for carcinogenesis. Angew. Chem. Int. Ed. 41, 445-448.

Senda, S., Wakayama, T., Arai, Y., et al. (2007). DNA methylation errors in cloned mice disappear with advancement of aging. Cloning Stem Cells 9, 293-302.

Stach, D., Schmitz, O.J., Stilgenbauer, S., et al. (2003). Capillary electrophoretic analysis of genomic DNA methylation levels. Nucleic Acids Res. 31, e2. 
Vignon, X., Chesné, P., Le Bourhis, D., et al. (1998). Developmental potential of bovine embryos reconstructed from enucleated matured oocytes fused with cultured somatic cells. C.R. Acad. Sci. Paris 321, 735-745.

Wells, D.N., Forsyth, J.T., McMillan, V., et al. (2004). The health of somatic cell cloned cattle and their offspring. Cloning Stem Cells 6, 101-110.

Wilmut, I. (2006). Are there any normal clones? Methods Mol. Biol. 348, 307-318.

Yang, X., Smith, S.L., Tian, X.C., et al. (2007a). Nuclear reprogramming of cloned embryos and its implications for therapeutic cloning. Nat. Genet. 39, 295-302.

Yang, X., Tian, X.C., Kubota, C., et al. (2007b). Assessment of meat and milk from cloned animals. Nat. Biotechnol. 25, 77-83.

Yang, J., Yang, S., Beaujean, N., et al. (2007c). Epigenetic marks in cloned rhesus monkey embryos: comparison with counterparts produced in vitro. Biol. Reprod. 76, 36-42.
Zakhartchenko, V., Durcova-Hills, G., Stojkovic, M., et al. (1999). Effects of serum starvation and re-cloning on the efficiency of nuclear transfer using bovine fetal fibroblasts. J. Reprod. Fertil. 115, 325-331.

Address correspondence to: Professor Stefan Hiendleder IS Davies Epigenetics and Genetics Group, School of Animal and Veterinary Science and Robinson Institute Research Centre for Reproductive Health The University of Adelaide Roseworthy Campus, Roseworthy, SA 5371, Australia

E-mail: Stefan.Hiendleder@adelaide.edu.au 
